CRYSTALLOGRAPHIC COMMUNICATIONS

ISSN 2056-9890

Received 8 November 2016

Accepted 12 November 2016

Edited by J. Simpson, University of Otago, New Zealand

Keywords: crystal structure; acaricide; insecticide; nematicide; oxamyl.

CCDC reference: 1516996

Supporting information: this article has supporting information at journals.iucr.org/e

\section{Crystal structure of oxamyl}

\author{
Eunjin Kwon, Ki-Min Park, ${ }^{*}$ Hyunjin Park and Tae Ho Kim*
}

Department of Chemistry (BK21 plus) and Research Institute of Natural Sciences, Gyeongsang National University, Jinju 52828, Republic of Korea. *Correspondence e-mail: kmpark@gnu.ac.kr, thkim@gnu.ac.kr

The title compound, $\mathrm{C}_{7} \mathrm{H}_{13} \mathrm{~N}_{3} \mathrm{O}_{3} \mathrm{~S}$ [systematic name: $(Z)$-methyl 2-dimethylamino- $N$-(methylcarbamoyloxy)-2-oxoethanimidothioate], is an oxime carbamate acaride, insecticide and nematicide. The asymmetric unit comprises two independent molecules, $A$ and $B$. The dihedral angles between the mean planes [r.m.s. deviations $=0.0017(A)$ and $0.0016 \AA(B)]$ of the acetamide and oxyimino groups are $88.80(8)^{\circ}$ for $A$ and $87.05(8)^{\circ}$ for $B$. In the crystal, $\mathrm{N} / \mathrm{C}-\mathrm{H} \cdots \mathrm{O}$ hydrogen bonds link adjacent molecules, forming chains along the $a$ axis. The chains are further linked by $\mathrm{C}-\mathrm{H} \cdots \mathrm{O}$ hydrogen bonds, resulting in a threedimensional network with alternating rows of $A$ and $B$ molecules in the $b c$ plane stacked along the $a$-axis direction. The structure was refined as an inversion twin with a final BASF parameter of $0.16(9)$.

\section{Chemical context}

Oxamyl $\quad[(N, N$-dimethyl-2-methylcarbamoyloximino-2-(dimethylsulfanyl)acetamide] is a carbamate compound used in a wide range of agricultural situations. It is systemic and active as an insecticide or a nematicide. It is used for the control of nematodes in vegetables, bananas, pineapple, peanuts, cotton, soya beans, tobacco, potatoes, sugar beet, and other crops. It is also used in India for controlling the growth of nematodes on vegetable crops (Mohammad et al., 2015; Agarwal et al., 2016). In addition, oxamyl was classified by the World Health Organization (WHO) as highly hazardous (class IB) (AlDabbas et al., 2014). Oxamyl can be integrated with horse manure, sesame-oil-cake, or Bacillus thuringiensis to improve eggplant growth response and reduce development of the nematode Meloidogyne incognita (Osman et al., 2009). Also, oxamyl has a very high water solubility $(280 \mathrm{~g} / \mathrm{L}$ at $298 \mathrm{~K})$ and low sorption solubility affinity to soils. As a result of these properties, oxamyl easily migrates into the water compartment (Mazellier et al., 2010). Herein, we report the molecular and crystal structure of oxamyl.

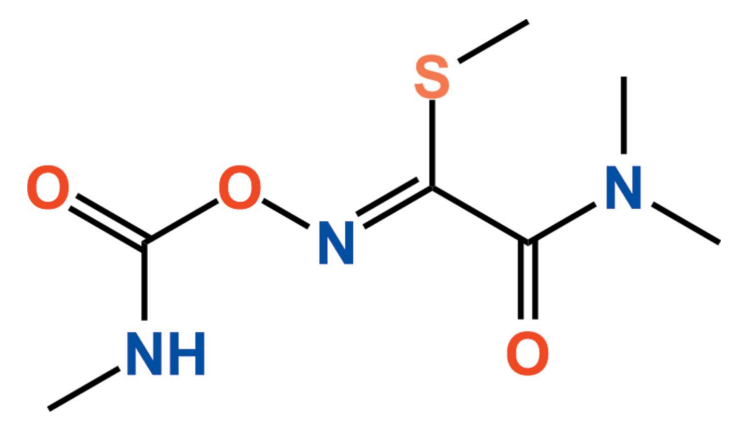


Table 1

Hydrogen-bond geometry $\left(\AA,^{\circ}\right)$.

\begin{tabular}{lllll}
\hline$D-\mathrm{H} \cdots A$ & $D-\mathrm{H}$ & $\mathrm{H} \cdots A$ & $D \cdots A$ & $D-\mathrm{H} \cdots A$ \\
\hline $\mathrm{N} 1-\mathrm{H} 1 N \cdots \mathrm{O} 1^{\text {i }}$ & 0.88 & 2.13 & $2.871(3)$ & 142 \\
$\mathrm{~N} 4-\mathrm{H} 4 N \cdots \mathrm{O} 4^{\text {ii }}$ & 0.88 & 2.04 & $2.794(3)$ & 142 \\
$\mathrm{C} 4-\mathrm{H} 4 B \cdots 6^{\text {iii }}$ & 0.98 & 2.54 & $3.075(4)$ & 114 \\
$\mathrm{C} 6-\mathrm{H} 6 B \cdots \mathrm{O} 2^{\text {iv }}$ & 0.98 & 2.60 & $3.518(4)$ & 156 \\
$\mathrm{C} 7-\mathrm{H} 7 B \cdots 1^{\text {iv }}$ & 0.98 & 2.52 & $3.431(4)$ & 155 \\
$\mathrm{C} 11-\mathrm{H} 11 B \cdots \mathrm{O} 3^{\text {v }}$ & 0.98 & 2.53 & $3.042(4)$ & 113 \\
$\mathrm{C} 13-\mathrm{H} 13 B \cdots \mathrm{O} 4^{\text {iv }}$ & 0.98 & 2.53 & $3.440(4)$ & 155 \\
$\mathrm{C} 14-\mathrm{H} 14 B \cdots 5^{\text {iv }}$ & 0.98 & 2.59 & $3.554(4)$ & 168
\end{tabular}

Symmetry codes: (i) $x-\frac{1}{2},-y, z$; (ii) $x-\frac{1}{2},-y+1, z$; (iii) $-x+1,-y+1, z+\frac{1}{2}$; (iv) $x-1, y, z ;(\mathrm{v})-x+1,-y, z-\frac{1}{2}$.

\section{Structural commentary}

The asymmetric unit of oxamyl comprises two independent molecules, $A$ and $B$ (Fig. 1). The compound consists of carbamate, acetamide, methylthio and oxyimino functional groups. The dihedral angles between the mean planes [r.m.s. deviations $=0.0017(A)$ and $0.0016 \AA(B)]$ of the acetamide and oxyimino groups are $88.80(8)$ for $A$ and $87.05(8)^{\circ}$ for $B$. All bond lengths and bond angles are normal and comparable to those observed in methomyl [systematic name: $(E)$-methyl $N$-(methylcarbamoyl)oxyethanimidothioate] which adopts similar crystal structure (Takusagawa \& Jacobson, 1977).

\section{Supramolecular features}

The crystal structure is stabilized by several $\mathrm{N}-\mathrm{H} \cdots \mathrm{O}$ and $\mathrm{C}-\mathrm{H} \cdots \mathrm{O}$ hydrogen bonds (Table 1 ). Adjacent $A$ molecules form intermolecular $\mathrm{N} 1-\mathrm{H} 1 N \cdots \mathrm{O} 1$ hydrogen bonds. In addition, $\mathrm{C} 6-\mathrm{H} 6 B \cdots \mathrm{O} 2$ and $\mathrm{C} 7-\mathrm{H} 7 B \cdots \mathrm{O} 1$ hydrogen bonds

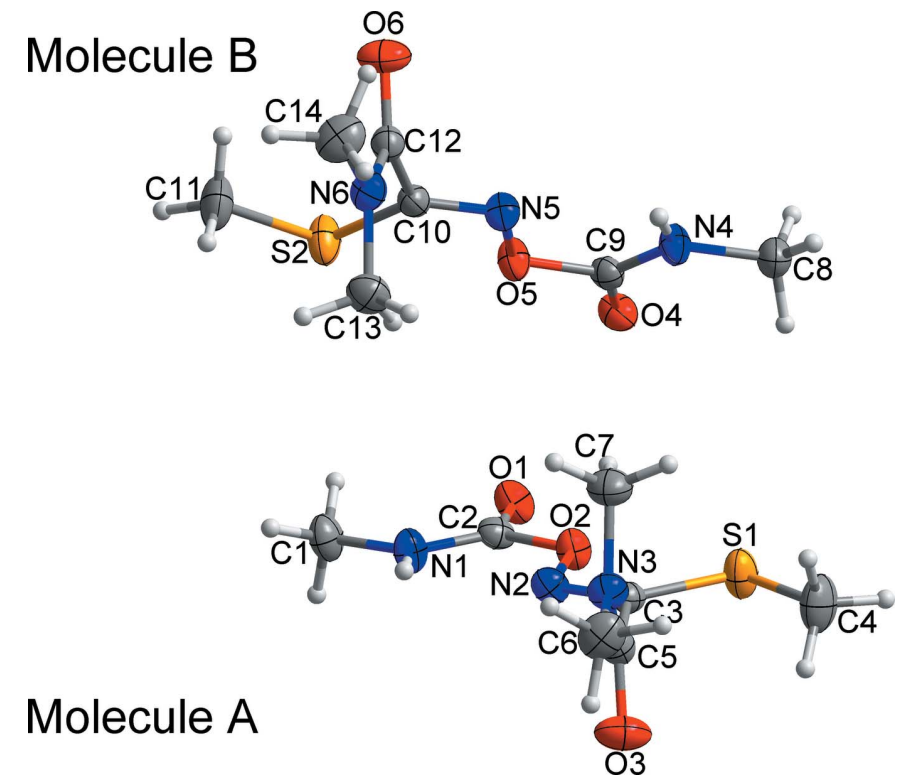

Figure 1

The asymmetric unit of the title compound, showing the atom-numbering scheme. Displacement ellipsoids are drawn at the $50 \%$ probability level. $\mathrm{H}$ atoms are shown as small spheres of arbitrary radius. between the carbamate and dimethylamine groups generate $R_{2}^{2}(8)$ inversion dimers. These contacts link the $A$ molecules into double chains along the $a$ axis. A closely similar situation obtains for the $B$ molecules, with intermolecular $\mathrm{N} 4-$ $\mathrm{H} 4 N \cdots \mathrm{O} 4$ hydrogen bonds together with $\mathrm{C} 13-\mathrm{H} 13 B \cdots \mathrm{O} 4$ and $\mathrm{C} 14-\mathrm{H} 14 B \cdots \mathrm{O} 5 R_{2}^{2}(8)$ inversion dimers also forming a double chain, this time solely of $B$ molecules, parallel to the one described previously, again along the $a$ axis, Fig. 2. The $A$ and $B$ double chains are further linked by $\mathrm{C} 4-\mathrm{H} 4 B \cdots \mathrm{O} 6$ and $\mathrm{C} 11-\mathrm{H} 11 B \cdots \mathrm{O} 3$ contacts, Table 1 , to give a three-dimensional network with alternating rows of $A$ and $B$ molecules in the $b c$ plane stacked along the $a$-axis direction, Fig. 3 .

\section{Synthesis and crystallization}

The title compound was purchased from Dr Ehrenstorfer $\mathrm{GmbH}$. Slow evaporation of its solution in $\mathrm{CH}_{3} \mathrm{OH}$ gave single crystals suitable for X-ray analysis.

\section{Refinement}

Crystal data, data collection and structure refinement details are summarized in Table 2. All C-bound $\mathrm{H}$ atoms were positioned geometrically [with $d(\mathrm{~N}-\mathrm{H})=0.88 \AA, U_{\text {iso }}=1.2 U_{\text {eq }}(\mathrm{C})$ for $\mathrm{N}-\mathrm{H}$ group, $U_{\text {iso }}=1.5 U_{\text {eq }}(\mathrm{C})$ for methyl group, $d(\mathrm{C}-\mathrm{H})=$ $0.98 \AA]$. The crystal studied was an inversion twin with a 0.84 (9):0.16 (9) domain ratio.

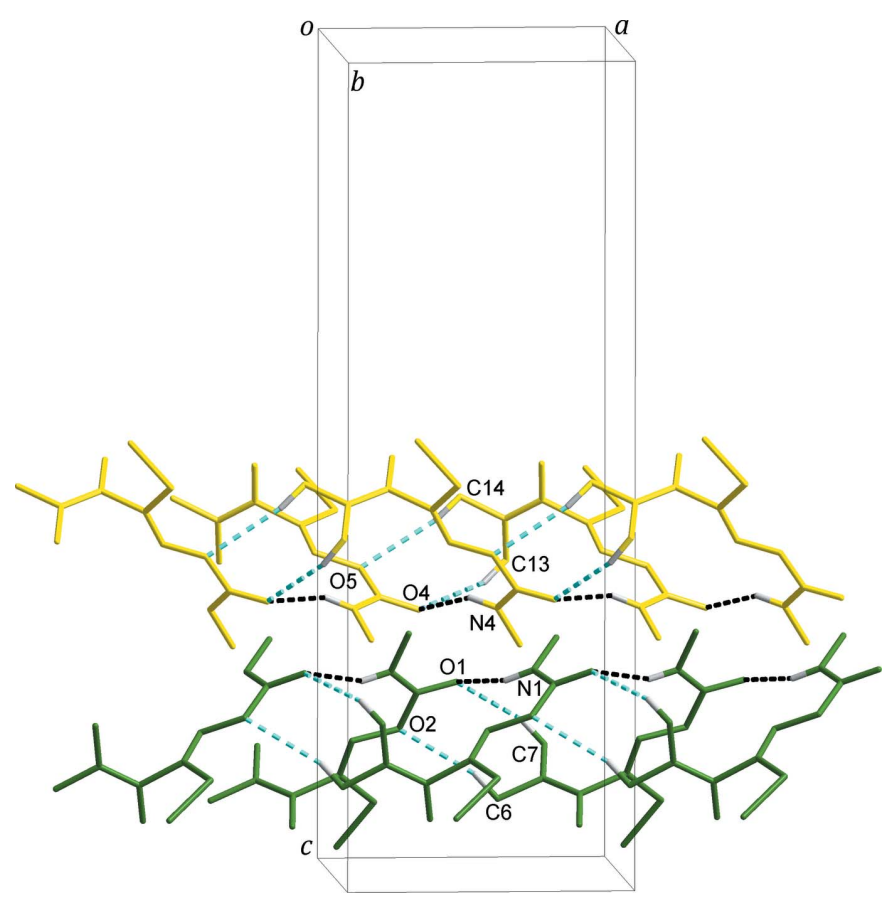

Figure 2

The double chains formed through intermolecular $\mathrm{N}-\mathrm{H} \cdots \mathrm{O}$ (black dashed lines) and $\mathrm{C}-\mathrm{H} \cdots \mathrm{O}$ (sky-blue dashed lines) hydrogen bonds. The $A$ and $B$ molecules are shown in green and yellow, respectively. $\mathrm{H}$ atoms not involved in intermolecular interactions have been omitted for clarity. 


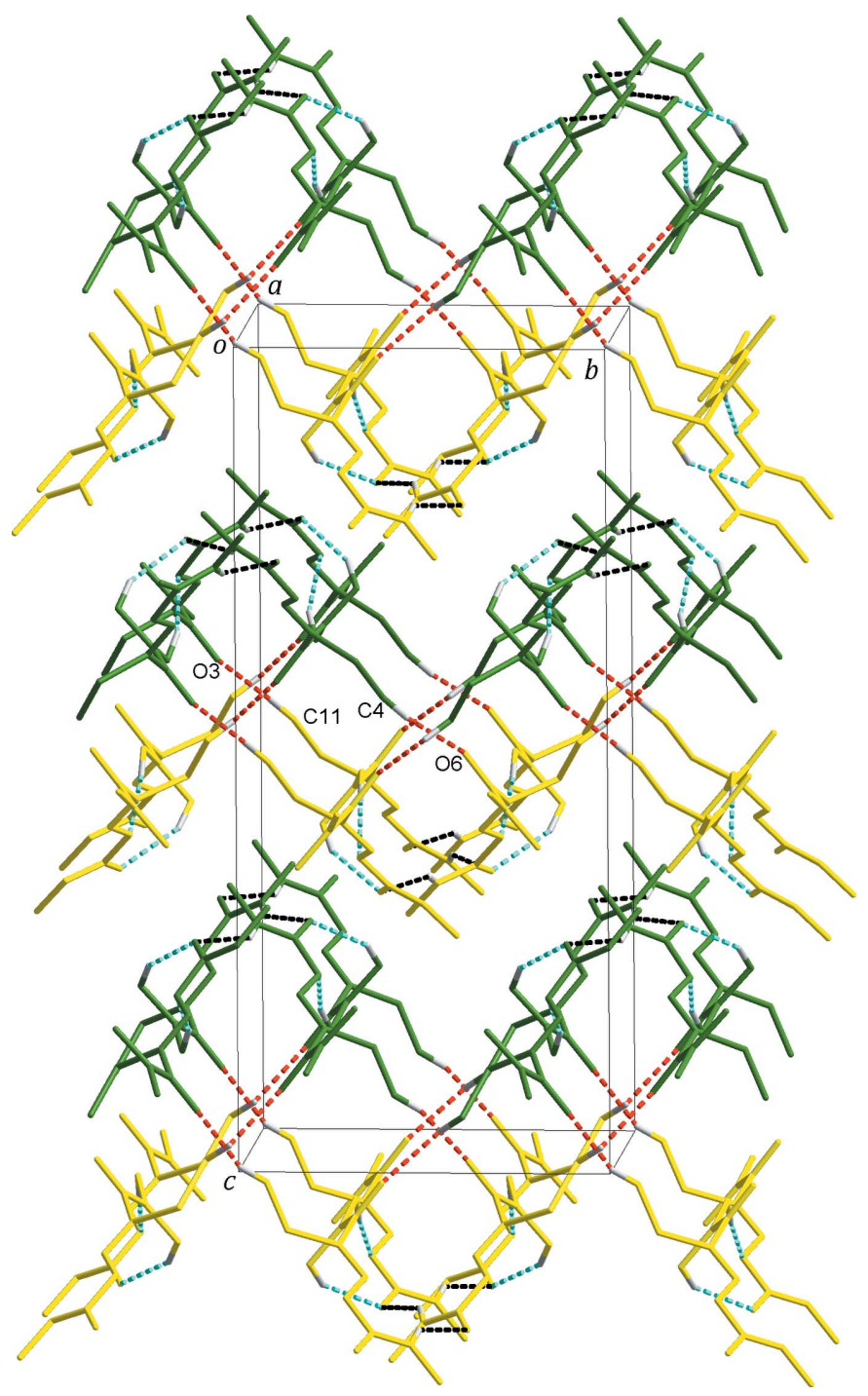

Figure 3

The three-dimensional network made up of molecules $A$ (green) and $B$ (yellow). Black dashed lines represent intermolecular $\mathrm{N}-\mathrm{H} \cdots \mathrm{O}$ hydrogen bonds. The $\mathrm{C}-\mathrm{H} \cdots \mathrm{O}$ hydrogen bonds are shown as sky-blue (between each molecule $A$ or $B$ ) and red (between molecules $A$ and $B$ ) dashed lines, respectively. $\mathrm{H}$ atoms not involved in intermolecular interactions have been omitted for clarity.

\section{Acknowledgements}

This research was supported by the Basic Science Program through the National Research Foundation of Korea (NRF) funded by the Ministry of Education, Science and Technology (No. 2015R1D1A4A01020317).
Table 2

Experimental details.

\begin{tabular}{|c|c|}
\hline \multicolumn{2}{|l|}{ Crystal data } \\
\hline Chemical formula & $\mathrm{C}_{7} \mathrm{H}_{13} \mathrm{~N}_{3} \mathrm{O}_{3} \mathrm{~S}$ \\
\hline$M_{\mathrm{r}}$ & 219.26 \\
\hline Crystal system, space group & Orthorhombic, $P c a 2_{1}$ \\
\hline Temperature $(\mathrm{K})$ & 173 \\
\hline$a, b, c(\AA)$ & $\begin{array}{l}8.3367(4), 10.7752(5) \\
24.1016(12)\end{array}$ \\
\hline$V\left(\AA^{3}\right)$ & $2165.04(18)$ \\
\hline$Z$ & 8 \\
\hline Radiation type & Mo $K \alpha$ \\
\hline$\mu\left(\mathrm{mm}^{-1}\right)$ & 0.29 \\
\hline Crystal size $(\mathrm{mm})$ & $0.50 \times 0.14 \times 0.11$ \\
\hline \multicolumn{2}{|l|}{ Data collection } \\
\hline Diffractometer & Bruker APEXII CCD \\
\hline Absorption correction & $\begin{array}{l}\text { Multi-scan (SADABS; Bruker, } \\
\text { 2014) }\end{array}$ \\
\hline$T_{\min }, T_{\max }$ & $0.665,0.746$ \\
\hline $\begin{array}{l}\text { No. of measured, independent and } \\
\text { observed }[I>2 \sigma(I)] \text { reflections }\end{array}$ & $19300,5238,4655$ \\
\hline$R_{\text {int }}$ & 0.035 \\
\hline$(\sin \theta / \lambda)_{\max }\left(\AA^{-1}\right)$ & 0.667 \\
\hline \multicolumn{2}{|l|}{ Refinement } \\
\hline$R\left[F^{2}>2 \sigma\left(F^{2}\right)\right], w R\left(F^{2}\right), S$ & $0.037,0.090,1.04$ \\
\hline No. of reflections & 5238 \\
\hline No. of parameters & 262 \\
\hline No. of restraints & 1 \\
\hline $\mathrm{H}$-atom treatment & $\mathrm{H}$-atom parameters constrained \\
\hline$\Delta \rho_{\max }, \Delta \rho_{\min }\left(\mathrm{e} \AA^{-3}\right)$ & $0.21,-0.23$ \\
\hline Absolute structure & Refined as an inversion twin \\
\hline Absolute structure parameter & $0.16(9)$ \\
\hline
\end{tabular}

Computer programs: APEX2 and SAINT (Bruker, 2014), SHELXS97 and SHELXTL (Sheldrick, 2008), SHELXL2014 (Sheldrick, 2015), DIAMOND (Brandenburg, 2010) and publCIF (Westrip, 2010).

\section{References}

Agarwal, S., Sadeghi, N., Tyagi, I., Gupta, V. K. \& Fakhri, A. (2016). J. Colloid Interface Sci. 478, 430-438.

Al-Dabbas, M. M., Shaderma, A. M. \& Al-Antary, T. M. (2014). Life Sci. J. 11, 68-73.

Brandenburg, K. (2010). DIAMOND. Crystal Impact GbR, Bonn, Germany.

Bruker (2014). APEX2, SAINT and SADABS. Bruker AXS Inc., Madison, Wisconsin, USA.

Mazellier, P., Zamy, C. \& Sarakha, M. (2010). Environ. Chem. Lett. 8, 19-24.

Mohammad, S. G., Ahmed, S. M. \& Badawi, A. F. M. (2015). Desalination Water Treatment, 55, 2109-2120.

Osman, K. A., Al-Rehiayani, S. M., Al-Deghairi, M. A. \& Salama, A. K. (2009). Int. Biodeterior. Biodegradation, 63, 341-346.

Sheldrick, G. M. (2008). Acta Cryst. A64, 112-122.

Sheldrick, G. M. (2015). Acta Cryst. C71, 3-8.

Takusagawa, F. \& Jacobson, R. A. (1977). J. Agric. Food Chem. 25, 577-581.

Westrip, S. P. (2010). J. Appl. Cryst. 43, 920-925. 


\section{supporting information}

Acta Cryst. (2016). E72, 1816-1818 [https://doi.org/10.1107/S2056989016018168]

\section{Crystal structure of oxamyl}

\section{Eunjin Kwon, Ki-Min Park, Hyunjin Park and Tae Ho Kim}

\section{Computing details}

Data collection: APEX2 (Bruker, 2014); cell refinement: SAINT (Bruker, 2014); data reduction: SAINT (Bruker, 2014); program(s) used to solve structure: SHELXS97 (Sheldrick, 2008); program(s) used to refine structure: SHELXL2014 (Sheldrick, 2015); molecular graphics: DIAMOND (Brandenburg, 2010); software used to prepare material for publication: SHELXTL (Sheldrick, 2008) and publCIF (Westrip, 2010).

(Z)-[(Dimethylcarbamoyl) (methylsulfanyl)methylidene]amino $N$-methylcarbamate

\section{Crystal data}

$\mathrm{C}_{7} \mathrm{H}_{13} \mathrm{~N}_{3} \mathrm{O}_{3} \mathrm{~S}$

$M_{r}=219.26$

Orthorhombic, $P c a 2_{1}$

$a=8.3367(4) \AA$

$b=10.7752(5) \AA$

$c=24.1016(12) \AA$

$V=2165.04(18) \AA^{3}$

$Z=8$

$F(000)=928$

\section{Data collection}

Bruker APEXII CCD diffractometer

$\varphi$ and $\omega$ scans

Absorption correction: multi-scan

(SADABS; Bruker, 2014)

$T_{\min }=0.665, T_{\max }=0.746$

19300 measured reflections

\section{Refinement}

Refinement on $F^{2}$

Least-squares matrix: full

$R\left[F^{2}>2 \sigma\left(F^{2}\right)\right]=0.037$

$w R\left(F^{2}\right)=0.090$

$S=1.04$

5238 reflections

262 parameters

1 restraint
$D_{\mathrm{x}}=1.345 \mathrm{Mg} \mathrm{m}^{-3}$

Mo $K \alpha$ radiation, $\lambda=0.71073 \AA$

Cell parameters from 6335 reflections

$\theta=2.5-27.8^{\circ}$

$\mu=0.29 \mathrm{~mm}^{-1}$

$T=173 \mathrm{~K}$

Plate, colourless

$0.50 \times 0.14 \times 0.11 \mathrm{~mm}$

5238 independent reflections 4655 reflections with $I>2 \sigma(I)$

$R_{\text {int }}=0.035$

$\theta_{\text {max }}=28.3^{\circ}, \theta_{\min }=1.7^{\circ}$

$h=-11 \rightarrow 11$

$k=-14 \rightarrow 14$

$l=-32 \rightarrow 31$

Hydrogen site location: inferred from neighbouring sites

$\mathrm{H}$-atom parameters constrained

$w=1 /\left[\sigma^{2}\left(F_{\mathrm{o}}^{2}\right)+(0.0432 P)^{2}+0.3998 P\right]$

$$
\text { where } P=\left(F_{\mathrm{o}}^{2}+2 F_{\mathrm{c}}^{2}\right) / 3
$$

$(\Delta / \sigma)_{\max }<0.001$

$\Delta \rho_{\max }=0.21$ e $\AA^{-3}$

$\Delta \rho_{\min }=-0.23$ e $\AA^{-3}$

Absolute structure: Refined as an inversion twin Absolute structure parameter: 0.16 (9) 


\section{Special details}

Geometry. All esds (except the esd in the dihedral angle between two 1.s. planes) are estimated using the full covariance matrix. The cell esds are taken into account individually in the estimation of esds in distances, angles and torsion angles; correlations between esds in cell parameters are only used when they are defined by crystal symmetry. An approximate (isotropic) treatment of cell esds is used for estimating esds involving l.s. planes.

Refinement. Refined as a 2-component inversion twin.

Fractional atomic coordinates and isotropic or equivalent isotropic displacement parameters $\left(\hat{A}^{2}\right)$

\begin{tabular}{|c|c|c|c|c|}
\hline & $x$ & $y$ & $z$ & $U_{\text {iso }} * / U_{\text {eq }}$ \\
\hline $\mathrm{S} 1$ & $0.55678(10)$ & $0.32807(7)$ & $0.87717(3)$ & $0.0373(2)$ \\
\hline $\mathrm{S} 2$ & $0.97239(10)$ & $0.16264(7)$ & $0.54545(3)$ & $0.0376(2)$ \\
\hline O1 & $0.8636(3)$ & $0.13823(18)$ & $0.74085(9)$ & $0.0308(5)$ \\
\hline $\mathrm{O} 2$ & $0.6589(2)$ & $0.17413(17)$ & $0.79576(8)$ & $0.0270(4)$ \\
\hline $\mathrm{O} 3$ & $0.2966(3)$ & $0.0966(2)$ & $0.91570(10)$ & $0.0431(6)$ \\
\hline $\mathrm{O} 4$ & $1.2851(3)$ & $0.36802(18)$ & $0.67471(9)$ & $0.0307(5)$ \\
\hline O5 & $1.0771(3)$ & $0.32628(17)$ & $0.62210(9)$ & $0.0282(5)$ \\
\hline O6 & $0.7167(3)$ & $0.3997(2)$ & $0.50123(10)$ & $0.0403(6)$ \\
\hline N1 & 0.6479 & $0.0112(2)$ & $0.73350(10)$ & $0.0290(5)$ \\
\hline $\mathrm{H} 1 \mathrm{~N}$ & 0.5497 & -0.0037 & 0.7451 & $0.035^{*}$ \\
\hline $\mathrm{N} 2$ & 0.5009 & 0.1328 & $0.81132(10)$ & $0.0272(5)$ \\
\hline N3 & $0.1571(3)$ & $0.2143(2)$ & $0.85463(10)$ & $0.0303(6)$ \\
\hline N4 & $1.0729(3)$ & $0.4993(2)$ & $0.67852(10)$ & $0.0287(5)$ \\
\hline $\mathrm{H} 4 \mathrm{~N}$ & 0.9764 & 0.5151 & 0.6655 & $0.034^{*}$ \\
\hline N5 & $0.9217(3)$ & $0.3683(2)$ & $0.60490(10)$ & $0.0261(5)$ \\
\hline N6 & $0.5764(3)$ & $0.2808(2)$ & $0.56227(10)$ & $0.0291(6)$ \\
\hline $\mathrm{C} 1$ & $0.7188(4)$ & $-0.0672(3)$ & $0.69115(14)$ & $0.0348(7)$ \\
\hline $\mathrm{H} 1 \mathrm{~A}$ & 0.7844 & -0.0164 & 0.6662 & $0.052 *$ \\
\hline H1B & 0.6335 & -0.1079 & 0.6699 & $0.052 *$ \\
\hline $\mathrm{H} 1 \mathrm{C}$ & 0.7863 & -0.1304 & 0.7088 & $0.052 *$ \\
\hline $\mathrm{C} 2$ & 0.7298 & $0.1050(2)$ & $0.75477(12)$ & $0.0244(6)$ \\
\hline $\mathrm{C} 3$ & $0.4493(4)$ & 0.2044 & $0.84969(11)$ & $0.0242(6)$ \\
\hline $\mathrm{C} 4$ & $0.4197(5)$ & $0.3917(4)$ & $0.92805(16)$ & $0.0481(10)$ \\
\hline $\mathrm{H} 4 \mathrm{~A}$ & 0.3255 & 0.4260 & 0.9091 & $0.072 *$ \\
\hline $\mathrm{H} 4 \mathrm{~B}$ & 0.4736 & 0.4576 & 0.9490 & $0.072 *$ \\
\hline $\mathrm{H} 4 \mathrm{C}$ & 0.3858 & 0.3259 & 0.9536 & $0.072^{*}$ \\
\hline $\mathrm{C} 5$ & 0.2909 (3) & $0.1659(3)$ & $0.87589(13)$ & $0.0271(6)$ \\
\hline C6 & $0.0028(4)$ & $0.1835(4)$ & $0.88024(16)$ & $0.0398(8)$ \\
\hline H6A & 0.0171 & 0.1138 & 0.9059 & $0.060^{*}$ \\
\hline H6B & -0.0742 & 0.1604 & 0.8513 & $0.060 *$ \\
\hline $\mathrm{H} 6 \mathrm{C}$ & -0.0376 & 0.2557 & 0.9007 & $0.060 *$ \\
\hline $\mathrm{C} 7$ & $0.1526(4)$ & $0.3046(3)$ & $0.80973(14)$ & $0.0372(7)$ \\
\hline H7A & 0.1188 & 0.3853 & 0.8243 & $0.056 *$ \\
\hline $\mathrm{H} 7 \mathrm{~B}$ & 0.0764 & 0.2770 & 0.7813 & $0.056^{*}$ \\
\hline $\mathrm{H} 7 \mathrm{C}$ & 0.2597 & 0.3122 & 0.7933 & $0.056^{*}$ \\
\hline $\mathrm{C} 8$ & $1.1426(4)$ & $0.5816(3)$ & $0.71943(13)$ & $0.0348(7)$ \\
\hline H8A & 1.1834 & 0.5326 & 0.7507 & $0.052^{*}$ \\
\hline H8B & 1.0607 & 0.6396 & 0.7327 & $0.052 *$ \\
\hline
\end{tabular}




$\begin{array}{lllll}\text { H8C } & 1.2311 & 0.6281 & 0.7026 & 0.052^{*} \\ \text { C9 } & 1.1516(4) & 0.4014(2) & 0.66055(11) & 0.0242(6) \\ \text { C10 } & 0.8681(4) & 0.2936(2) & 0.56845(11) & 0.0240(6) \\ \text { C11 } & 0.8379(5) & 0.0982(3) & 0.49430(15) & 0.0462(9) \\ \text { H11A } & 0.7431 & 0.0641 & 0.5129 & 0.069^{*} \\ \text { H11B } & 0.8928 & 0.0320 & 0.4739 & 0.069^{*} \\ \text { H11C } & 0.8049 & 0.1635 & 0.4684 & 0.069^{*} \\ \text { C12 } & 0.7109(4) & 0.3295(2) & 0.54159(13) & 0.0267(6) \\ \text { C13 } & 0.5720(4) & 0.1938(3) & 0.60790(14) & 0.0366(7) \\ \text { H13A } & 0.5364 & 0.1126 & 0.5944 & 0.055^{*} \\ \text { H13B } & 0.4972 & 0.2238 & 0.6363 & 0.055^{*} \\ \text { H13C } & 0.6795 & 0.1861 & 0.6240 & 0.055^{*} \\ \text { C14 } & 0.4231(4) & 0.3106(4) & 0.53622(15) & 0.0420(8) \\ \text { H14A } & 0.4301 & 0.3922 & 0.5184 & 0.063^{*} \\ \text { H14B } & 0.3386 & 0.3120 & 0.5645 & 0.063^{*} \\ \text { H14C } & 0.3976 & 0.2476 & 0.5083 & 0.063^{*}\end{array}$

Atomic displacement parameters $\left(\AA^{2}\right)$

\begin{tabular}{|c|c|c|c|c|c|c|}
\hline & $U^{11}$ & $U^{22}$ & $U^{33}$ & $U^{12}$ & $U^{13}$ & $U^{23}$ \\
\hline S1 & $0.0356(4)$ & $0.0368(4)$ & $0.0393(4)$ & $-0.0072(3)$ & $0.0091(4)$ & $-0.0137(4)$ \\
\hline S2 & $0.0352(4)$ & $0.0330(4)$ & $0.0447(5)$ & $0.0083(3)$ & $-0.0103(4)$ & -0.0155 \\
\hline $\mathrm{O} 1$ & $0.0210(10)$ & $0.0284(10)$ & $0.0428(12)$ & $-0.0018(9)$ & $0.0060(9)$ & $-0.0027(9)$ \\
\hline $\mathrm{O} 2$ & $0.0212(10)$ & $0.0271(10)$ & $0.0325(11)$ & $-0.0017(8)$ & $0.0047(9)$ & $-0.0055(8)$ \\
\hline $\mathrm{O} 3$ & $0.0392(14)$ & $0.0478(13)$ & $0.0423(13)$ & $0.0036(11)$ & $0.0085(11)$ & $0.0196(11)$ \\
\hline $\mathrm{O} 4$ & $0.0246(11)$ & $0.0275(10)$ & $0.0401(12)$ & $-0.0007(9)$ & $-0.0058(10)$ & $0.0029(9)$ \\
\hline O5 & $0.0211(10)$ & $0.0257(10)$ & $0.0379(12)$ & $0.0023(8)$ & $-0.0061(9)$ & $-0.0072(9)$ \\
\hline O6 & $0.0342(13)$ & $0.0476(12)$ & $0.0393(12)$ & $0.0008(11)$ & $-0.0035(10)$ & $0.0185(11)$ \\
\hline N1 & $0.0227(12)$ & $0.0282(11)$ & $0.0360(13)$ & $-0.0003(10)$ & $0.0061(10)$ & $-0.0077(10)$ \\
\hline $\mathrm{N} 2$ & $0.0233(13)$ & $0.0265(12)$ & $0.0318(13)$ & $-0.0011(11)$ & $0.0054(11)$ & $0.0001(10)$ \\
\hline N3 & $0.0269(13)$ & $0.0338(13)$ & $0.0303(13)$ & $-0.0022(11)$ & $0.0031(11)$ & $0.0026(10)$ \\
\hline N4 & $0.0259(12)$ & $0.0261(11)$ & $0.0343(12)$ & $0.0015(10)$ & $-0.0078(10)$ & $-0.0065(10)$ \\
\hline N5 & $0.0204(12)$ & $0.0268(11)$ & $0.0310(13)$ & $0.0025(10)$ & $-0.0039(10)$ & $-0.0028(10)$ \\
\hline N6 & $0.0242(13)$ & $0.0338(12)$ & $0.0293(13)$ & $-0.0019(10)$ & $-0.0014(10)$ & $-0.0019(10)$ \\
\hline $\mathrm{C} 1$ & $0.0351(17)$ & $0.0295(15)$ & $0.0398(17)$ & $0.0018(13)$ & $0.0061(14)$ & $-0.0103(12)$ \\
\hline $\mathrm{C} 2$ & $0.0232(15)$ & $0.0219(12)$ & $0.0281(14)$ & $0.0057(11)$ & $0.0031(12)$ & $0.0023(11)$ \\
\hline $\mathrm{C} 3$ & $0.0265(15)$ & $0.0235(13)$ & $0.0225(13)$ & $0.0020(12)$ & $0.0005(11)$ & $0.0021(11)$ \\
\hline $\mathrm{C} 4$ & $0.047(2)$ & $0.052(2)$ & $0.045(2)$ & $0.0018(18)$ & $0.0094(18)$ & $-0.0225(17)$ \\
\hline $\mathrm{C} 5$ & $0.0276(15)$ & $0.0256(13)$ & $0.0282(14)$ & $-0.0014(11)$ & $0.0051(13)$ & $-0.0014(12)$ \\
\hline C6 & $0.0255(15)$ & $0.055(2)$ & $0.0388(17)$ & $-0.0041(15)$ & $0.0053(16)$ & $-0.0005(16)$ \\
\hline $\mathrm{C} 7$ & $0.0364(18)$ & $0.0385(17)$ & $0.0368(17)$ & $0.0014(14)$ & $-0.0027(15)$ & $0.0072(14)$ \\
\hline $\mathrm{C} 8$ & $0.0439(19)$ & $0.0293(14)$ & $0.0311(15)$ & $-0.0019(14)$ & $-0.0055(14)$ & $-0.0050(12)$ \\
\hline C9 & $0.0236(14)$ & $0.0225(13)$ & $0.0266(13)$ & $-0.0036(11)$ & $-0.0002(12)$ & $0.0020(11)$ \\
\hline $\mathrm{C} 10$ & $0.0262(15)$ & $0.0210(12)$ & $0.0249(13)$ & $0.0001(12)$ & $0.0008(11)$ & $0.0001(11)$ \\
\hline $\mathrm{C} 11$ & $0.048(2)$ & $0.0461(19)$ & $0.045(2)$ & $0.0003(17)$ & $-0.0105(17)$ & $-0.0213(16)$ \\
\hline $\mathrm{C} 12$ & $0.0277(15)$ & $0.0253(12)$ & $0.0271(14)$ & $0.0005(11)$ & $-0.0025(13)$ & $-0.0024(12)$ \\
\hline $\mathrm{C} 13$ & $0.0346(18)$ & $0.0368(16)$ & $0.0383(18)$ & $-0.0050(14)$ & $0.0050(14)$ & $0.0040(14)$ \\
\hline $\mathrm{C} 14$ & $0.0240(15)$ & $0.057(2)$ & $0.045(2)$ & $0.0030(15)$ & $-0.0041(15)$ & $0.0001(16)$ \\
\hline
\end{tabular}


Geometric parameters $\left(\AA,{ }^{\circ}\right)$

\begin{tabular}{|c|c|c|c|}
\hline $\mathrm{S} 1-\mathrm{C} 3$ & $1.737(3)$ & $\mathrm{C} 1-\mathrm{H} 1 \mathrm{~A}$ & 0.9800 \\
\hline $\mathrm{S} 1-\mathrm{C} 4$ & $1.811(3)$ & $\mathrm{C} 1-\mathrm{H} 1 \mathrm{~B}$ & 0.9800 \\
\hline $\mathrm{S} 2-\mathrm{C} 10$ & $1.748(3)$ & $\mathrm{C} 1-\mathrm{H} 1 \mathrm{C}$ & 0.9800 \\
\hline $\mathrm{S} 2-\mathrm{C} 11$ & $1.805(3)$ & $\mathrm{C} 3-\mathrm{C} 5$ & $1.521(4)$ \\
\hline $\mathrm{O} 1-\mathrm{C} 2$ & $1.218(4)$ & $\mathrm{C} 4-\mathrm{H} 4 \mathrm{~A}$ & 0.9800 \\
\hline $\mathrm{O} 2-\mathrm{C} 2$ & $1.371(3)$ & $\mathrm{C} 4-\mathrm{H} 4 \mathrm{~B}$ & 0.9800 \\
\hline $\mathrm{O} 2-\mathrm{N} 2$ & $1.440(3)$ & $\mathrm{C} 4-\mathrm{H} 4 \mathrm{C}$ & 0.9800 \\
\hline $\mathrm{O} 3-\mathrm{C} 5$ & $1.216(4)$ & C6-H6A & 0.9800 \\
\hline $\mathrm{O} 4-\mathrm{C} 9$ & $1.219(4)$ & $\mathrm{C} 6-\mathrm{H} 6 \mathrm{~B}$ & 0.9800 \\
\hline $\mathrm{O} 5-\mathrm{C} 9$ & $1.378(3)$ & $\mathrm{C} 6-\mathrm{H} 6 \mathrm{C}$ & 0.9800 \\
\hline $\mathrm{O} 5-\mathrm{N} 5$ & $1.434(3)$ & $\mathrm{C} 7-\mathrm{H} 7 \mathrm{~A}$ & 0.9800 \\
\hline $\mathrm{O} 6-\mathrm{C} 12$ & $1.233(4)$ & $\mathrm{C} 7-\mathrm{H} 7 \mathrm{~B}$ & 0.9800 \\
\hline $\mathrm{N} 1-\mathrm{C} 2$ & $1.323(4)$ & $\mathrm{C} 7-\mathrm{H} 7 \mathrm{C}$ & 0.9800 \\
\hline $\mathrm{N} 1-\mathrm{C} 1$ & $1.451(4)$ & $\mathrm{C} 8-\mathrm{H} 8 \mathrm{~A}$ & 0.9800 \\
\hline $\mathrm{N} 1-\mathrm{H} 1 \mathrm{~N}$ & 0.8800 & $\mathrm{C} 8-\mathrm{H} 8 \mathrm{~B}$ & 0.9800 \\
\hline $\mathrm{N} 2-\mathrm{C} 3$ & $1.279(4)$ & $\mathrm{C} 8-\mathrm{H} 8 \mathrm{C}$ & 0.9800 \\
\hline $\mathrm{N} 3-\mathrm{C} 5$ & $1.334(4)$ & $\mathrm{C} 10-\mathrm{C} 12$ & $1.512(4)$ \\
\hline $\mathrm{N} 3-\mathrm{C} 7$ & $1.455(4)$ & C11-H11A & 0.9800 \\
\hline N3-C6 & $1.464(4)$ & C11-H11B & 0.9800 \\
\hline $\mathrm{N} 4-\mathrm{C} 9$ & $1.315(4)$ & $\mathrm{C} 11-\mathrm{H} 11 \mathrm{C}$ & 0.9800 \\
\hline $\mathrm{N} 4-\mathrm{C} 8$ & $1.448(4)$ & $\mathrm{C} 13-\mathrm{H} 13 \mathrm{~A}$ & 0.9800 \\
\hline $\mathrm{N} 4-\mathrm{H} 4 \mathrm{~N}$ & 0.8800 & C13-H13B & 0.9800 \\
\hline $\mathrm{N} 5-\mathrm{C} 10$ & $1.272(4)$ & $\mathrm{C} 13-\mathrm{H} 13 \mathrm{C}$ & 0.9800 \\
\hline N6-C12 & $1.334(4)$ & $\mathrm{C} 14-\mathrm{H} 14 \mathrm{~A}$ & 0.9800 \\
\hline N6-C13 & $1.445(4)$ & C14-H14B & 0.9800 \\
\hline $\mathrm{N} 6-\mathrm{C} 14$ & $1.460(4)$ & $\mathrm{C} 14-\mathrm{H} 14 \mathrm{C}$ & 0.9800 \\
\hline $\mathrm{C} 3-\mathrm{S} 1-\mathrm{C} 4$ & $102.89(16)$ & $\mathrm{N} 3-\mathrm{C} 6-\mathrm{H} 6 \mathrm{C}$ & 109.5 \\
\hline $\mathrm{C} 10-\mathrm{S} 2-\mathrm{C} 11$ & $102.61(16)$ & $\mathrm{H} 6 \mathrm{~A}-\mathrm{C} 6-\mathrm{H} 6 \mathrm{C}$ & 109.5 \\
\hline $\mathrm{C} 2-\mathrm{O} 2-\mathrm{N} 2$ & $114.5(2)$ & $\mathrm{H} 6 \mathrm{~B}-\mathrm{C} 6-\mathrm{H} 6 \mathrm{C}$ & 109.5 \\
\hline $\mathrm{C} 9-\mathrm{O} 5-\mathrm{N} 5$ & $114.6(2)$ & $\mathrm{N} 3-\mathrm{C} 7-\mathrm{H} 7 \mathrm{~A}$ & 109.5 \\
\hline $\mathrm{C} 2-\mathrm{N} 1-\mathrm{C} 1$ & $120.5(2)$ & $\mathrm{N} 3-\mathrm{C} 7-\mathrm{H} 7 \mathrm{~B}$ & 109.5 \\
\hline $\mathrm{C} 2-\mathrm{N} 1-\mathrm{H} 1 \mathrm{~N}$ & 119.8 & $\mathrm{H} 7 \mathrm{~A}-\mathrm{C} 7-\mathrm{H} 7 \mathrm{~B}$ & 109.5 \\
\hline $\mathrm{C} 1-\mathrm{N} 1-\mathrm{H} 1 \mathrm{~N}$ & 119.8 & $\mathrm{~N} 3-\mathrm{C} 7-\mathrm{H} 7 \mathrm{C}$ & 109.5 \\
\hline $\mathrm{C} 3-\mathrm{N} 2-\mathrm{O} 2$ & $108.1(2)$ & $\mathrm{H} 7 \mathrm{~A}-\mathrm{C} 7-\mathrm{H} 7 \mathrm{C}$ & 109.5 \\
\hline $\mathrm{C} 5-\mathrm{N} 3-\mathrm{C} 7$ & $124.6(3)$ & $\mathrm{H} 7 \mathrm{~B}-\mathrm{C} 7-\mathrm{H} 7 \mathrm{C}$ & 109.5 \\
\hline $\mathrm{C} 5-\mathrm{N} 3-\mathrm{C} 6$ & $118.9(3)$ & $\mathrm{N} 4-\mathrm{C} 8-\mathrm{H} 8 \mathrm{~A}$ & 109.5 \\
\hline $\mathrm{C} 7-\mathrm{N} 3-\mathrm{C} 6$ & $116.3(3)$ & $\mathrm{N} 4-\mathrm{C} 8-\mathrm{H} 8 \mathrm{~B}$ & 109.5 \\
\hline $\mathrm{C} 9-\mathrm{N} 4-\mathrm{C} 8$ & $121.0(3)$ & $\mathrm{H} 8 \mathrm{~A}-\mathrm{C} 8-\mathrm{H} 8 \mathrm{~B}$ & 109.5 \\
\hline $\mathrm{C} 9-\mathrm{N} 4-\mathrm{H} 4 \mathrm{~N}$ & 119.5 & $\mathrm{~N} 4-\mathrm{C} 8-\mathrm{H} 8 \mathrm{C}$ & 109.5 \\
\hline $\mathrm{C} 8-\mathrm{N} 4-\mathrm{H} 4 \mathrm{~N}$ & 119.5 & $\mathrm{H} 8 \mathrm{~A}-\mathrm{C} 8-\mathrm{H} 8 \mathrm{C}$ & 109.5 \\
\hline $\mathrm{C} 10-\mathrm{N} 5-\mathrm{O} 5$ & $108.5(2)$ & $\mathrm{H} 8 \mathrm{~B}-\mathrm{C} 8-\mathrm{H} 8 \mathrm{C}$ & 109.5 \\
\hline $\mathrm{C} 12-\mathrm{N} 6-\mathrm{C} 13$ & $124.1(3)$ & $\mathrm{O} 4-\mathrm{C} 9-\mathrm{N} 4$ & $126.9(3)$ \\
\hline $\mathrm{C} 12-\mathrm{N} 6-\mathrm{C} 14$ & $119.2(3)$ & $\mathrm{O} 4-\mathrm{C} 9-\mathrm{O} 5$ & $115.2(2)$ \\
\hline $\mathrm{C} 13-\mathrm{N} 6-\mathrm{C} 14$ & $116.6(3)$ & $\mathrm{N} 4-\mathrm{C} 9-\mathrm{O} 5$ & $117.9(3)$ \\
\hline $\mathrm{N} 1-\mathrm{C} 1-\mathrm{H} 1 \mathrm{~A}$ & 109.5 & $\mathrm{~N} 5-\mathrm{C} 10-\mathrm{C} 12$ & $116.0(2)$ \\
\hline
\end{tabular}




\begin{tabular}{|c|c|c|c|}
\hline $\mathrm{N} 1-\mathrm{C} 1-\mathrm{H} 1 \mathrm{~B}$ & 109.5 & $\mathrm{~N} 5-\mathrm{C} 10-\mathrm{S} 2$ & $123.7(2)$ \\
\hline $\mathrm{H} 1 \mathrm{~A}-\mathrm{C} 1-\mathrm{H} 1 \mathrm{~B}$ & 109.5 & $\mathrm{C} 12-\mathrm{C} 10-\mathrm{S} 2$ & $120.1(2)$ \\
\hline $\mathrm{N} 1-\mathrm{C} 1-\mathrm{H} 1 \mathrm{C}$ & 109.5 & $\mathrm{~S} 2-\mathrm{C} 11-\mathrm{H} 11 \mathrm{~A}$ & 109.5 \\
\hline $\mathrm{H} 1 \mathrm{~A}-\mathrm{C} 1-\mathrm{H} 1 \mathrm{C}$ & 109.5 & $\mathrm{~S} 2-\mathrm{C} 11-\mathrm{H} 11 \mathrm{~B}$ & 109.5 \\
\hline $\mathrm{H} 1 \mathrm{~B}-\mathrm{C} 1-\mathrm{H} 1 \mathrm{C}$ & 109.5 & $\mathrm{H} 11 \mathrm{~A}-\mathrm{C} 11-\mathrm{H} 11 \mathrm{~B}$ & 109.5 \\
\hline $\mathrm{O} 1-\mathrm{C} 2-\mathrm{N} 1$ & $126.2(3)$ & $\mathrm{S} 2-\mathrm{C} 11-\mathrm{H} 11 \mathrm{C}$ & 109.5 \\
\hline $\mathrm{O} 1-\mathrm{C} 2-\mathrm{O} 2$ & $115.7(2)$ & $\mathrm{H} 11 \mathrm{~A}-\mathrm{C} 11-\mathrm{H} 11 \mathrm{C}$ & 109.5 \\
\hline $\mathrm{N} 1-\mathrm{C} 2-\mathrm{O} 2$ & $118.1(3)$ & $\mathrm{H} 11 \mathrm{~B}-\mathrm{C} 11-\mathrm{H} 11 \mathrm{C}$ & 109.5 \\
\hline $\mathrm{N} 2-\mathrm{C} 3-\mathrm{C} 5$ & $115.3(2)$ & $\mathrm{O} 6-\mathrm{C} 12-\mathrm{N} 6$ & $124.7(3)$ \\
\hline $\mathrm{N} 2-\mathrm{C} 3-\mathrm{S} 1$ & $124.4(2)$ & $\mathrm{O} 6-\mathrm{C} 12-\mathrm{C} 10$ & $117.4(3)$ \\
\hline $\mathrm{C} 5-\mathrm{C} 3-\mathrm{S} 1$ & $119.9(2)$ & $\mathrm{N} 6-\mathrm{C} 12-\mathrm{C} 10$ & $117.9(3)$ \\
\hline $\mathrm{S} 1-\mathrm{C} 4-\mathrm{H} 4 \mathrm{~A}$ & 109.5 & $\mathrm{~N} 6-\mathrm{C} 13-\mathrm{H} 13 \mathrm{~A}$ & 109.5 \\
\hline $\mathrm{S} 1-\mathrm{C} 4-\mathrm{H} 4 \mathrm{~B}$ & 109.5 & $\mathrm{~N} 6-\mathrm{C} 13-\mathrm{H} 13 \mathrm{~B}$ & 109.5 \\
\hline $\mathrm{H} 4 \mathrm{~A}-\mathrm{C} 4-\mathrm{H} 4 \mathrm{~B}$ & 109.5 & $\mathrm{H} 13 \mathrm{~A}-\mathrm{C} 13-\mathrm{H} 13 \mathrm{~B}$ & 109.5 \\
\hline $\mathrm{S} 1-\mathrm{C} 4-\mathrm{H} 4 \mathrm{C}$ & 109.5 & $\mathrm{~N} 6-\mathrm{C} 13-\mathrm{H} 13 \mathrm{C}$ & 109.5 \\
\hline $\mathrm{H} 4 \mathrm{~A}-\mathrm{C} 4-\mathrm{H} 4 \mathrm{C}$ & 109.5 & $\mathrm{H} 13 \mathrm{~A}-\mathrm{C} 13-\mathrm{H} 13 \mathrm{C}$ & 109.5 \\
\hline $\mathrm{H} 4 \mathrm{~B}-\mathrm{C} 4-\mathrm{H} 4 \mathrm{C}$ & 109.5 & $\mathrm{H} 13 \mathrm{~B}-\mathrm{C} 13-\mathrm{H} 13 \mathrm{C}$ & 109.5 \\
\hline $\mathrm{O} 3-\mathrm{C} 5-\mathrm{N} 3$ & $125.1(3)$ & $\mathrm{N} 6-\mathrm{C} 14-\mathrm{H} 14 \mathrm{~A}$ & 109.5 \\
\hline $\mathrm{O} 3-\mathrm{C} 5-\mathrm{C} 3$ & $117.4(3)$ & N6-C14-H14B & 109.5 \\
\hline $\mathrm{N} 3-\mathrm{C} 5-\mathrm{C} 3$ & $117.4(3)$ & $\mathrm{H} 14 \mathrm{~A}-\mathrm{C} 14-\mathrm{H} 14 \mathrm{~B}$ & 109.5 \\
\hline $\mathrm{N} 3-\mathrm{C} 6-\mathrm{H} 6 \mathrm{~A}$ & 109.5 & $\mathrm{~N} 6-\mathrm{C} 14-\mathrm{H} 14 \mathrm{C}$ & 109.5 \\
\hline $\mathrm{N} 3-\mathrm{C} 6-\mathrm{H} 6 \mathrm{~B}$ & 109.5 & $\mathrm{H} 14 \mathrm{~A}-\mathrm{C} 14-\mathrm{H} 14 \mathrm{C}$ & 109.5 \\
\hline $\mathrm{H} 6 \mathrm{~A}-\mathrm{C} 6-\mathrm{H} 6 \mathrm{~B}$ & 109.5 & $\mathrm{H} 14 \mathrm{~B}-\mathrm{C} 14-\mathrm{H} 14 \mathrm{C}$ & 109.5 \\
\hline $\mathrm{C} 2-\mathrm{O} 2-\mathrm{N} 2-\mathrm{C} 3$ & $179.8(2)$ & $\mathrm{S} 1-\mathrm{C} 3-\mathrm{C} 5-\mathrm{N} 3$ & $93.6(3)$ \\
\hline $\mathrm{C} 9-\mathrm{O} 5-\mathrm{N} 5-\mathrm{C} 10$ & $178.1(2)$ & $\mathrm{C} 8-\mathrm{N} 4-\mathrm{C} 9-\mathrm{O} 4$ & $0.0(5)$ \\
\hline $\mathrm{C} 1-\mathrm{N} 1-\mathrm{C} 2-\mathrm{O} 1$ & $3.0(5)$ & $\mathrm{C} 8-\mathrm{N} 4-\mathrm{C} 9-\mathrm{O} 5$ & $179.2(2)$ \\
\hline $\mathrm{C} 1-\mathrm{N} 1-\mathrm{C} 2-\mathrm{O} 2$ & $-178.2(3)$ & $\mathrm{N} 5-\mathrm{O} 5-\mathrm{C} 9-\mathrm{O} 4$ & $-178.6(2)$ \\
\hline $\mathrm{N} 2-\mathrm{O} 2-\mathrm{C} 2-\mathrm{O} 1$ & $179.9(2)$ & $\mathrm{N} 5-\mathrm{O} 5-\mathrm{C} 9-\mathrm{N} 4$ & $2.1(3)$ \\
\hline $\mathrm{N} 2-\mathrm{O} 2-\mathrm{C} 2-\mathrm{N} 1$ & $1.0(3)$ & $\mathrm{O} 5-\mathrm{N} 5-\mathrm{C} 10-\mathrm{C} 12$ & $-174.7(2)$ \\
\hline $\mathrm{O} 2-\mathrm{N} 2-\mathrm{C} 3-\mathrm{C} 5$ & $-173.4(2)$ & $\mathrm{O} 5-\mathrm{N} 5-\mathrm{C} 10-\mathrm{S} 2$ & $0.4(3)$ \\
\hline $\mathrm{O} 2-\mathrm{N} 2-\mathrm{C} 3-\mathrm{S} 1$ & $-0.5(3)$ & $\mathrm{C} 11-\mathrm{S} 2-\mathrm{C} 10-\mathrm{N} 5$ & $-179.2(3)$ \\
\hline $\mathrm{C} 4-\mathrm{S} 1-\mathrm{C} 3-\mathrm{N} 2$ & $179.3(3)$ & $\mathrm{C} 11-\mathrm{S} 2-\mathrm{C} 10-\mathrm{C} 12$ & $-4.2(3)$ \\
\hline $\mathrm{C} 4-\mathrm{S} 1-\mathrm{C} 3-\mathrm{C} 5$ & $-8.0(3)$ & $\mathrm{C} 13-\mathrm{N} 6-\mathrm{C} 12-\mathrm{O} 6$ & $176.8(3)$ \\
\hline $\mathrm{C} 7-\mathrm{N} 3-\mathrm{C} 5-\mathrm{O} 3$ & $175.0(3)$ & $\mathrm{C} 14-\mathrm{N} 6-\mathrm{C} 12-\mathrm{O} 6$ & $0.1(4)$ \\
\hline $\mathrm{C} 6-\mathrm{N} 3-\mathrm{C} 5-\mathrm{O} 3$ & $-0.1(5)$ & $\mathrm{C} 13-\mathrm{N} 6-\mathrm{C} 12-\mathrm{C} 10$ & $-1.4(4)$ \\
\hline $\mathrm{C} 7-\mathrm{N} 3-\mathrm{C} 5-\mathrm{C} 3$ & $-2.4(4)$ & $\mathrm{C} 14-\mathrm{N} 6-\mathrm{C} 12-\mathrm{C} 10$ & $-178.1(3)$ \\
\hline $\mathrm{C} 6-\mathrm{N} 3-\mathrm{C} 5-\mathrm{C} 3$ & $-177.5(3)$ & $\mathrm{N} 5-\mathrm{C} 10-\mathrm{C} 12-\mathrm{O} 6$ & $85.8(3)$ \\
\hline $\mathrm{N} 2-\mathrm{C} 3-\mathrm{C} 5-\mathrm{O} 3$ & $89.3(3)$ & $\mathrm{S} 2-\mathrm{C} 10-\mathrm{C} 12-\mathrm{O} 6$ & $-89.5(3)$ \\
\hline $\mathrm{S} 1-\mathrm{C} 3-\mathrm{C} 5-\mathrm{O} 3$ & $-84.0(3)$ & $\mathrm{N} 5-\mathrm{C} 10-\mathrm{C} 12-\mathrm{N} 6$ & $-95.9(3)$ \\
\hline $\mathrm{N} 2-\mathrm{C} 3-\mathrm{C} 5-\mathrm{N} 3$ & $-93.1(3)$ & $\mathrm{S} 2-\mathrm{C} 10-\mathrm{C} 12-\mathrm{N} 6$ & $88.8(3)$ \\
\hline
\end{tabular}

Hydrogen-bond geometry $\left(A,{ }^{\circ}\right)$

\begin{tabular}{lllll}
\hline$D-\mathrm{H} \cdots A$ & $D-\mathrm{H}$ & $\mathrm{H} \cdots A$ & $D \cdots A$ & $D-\mathrm{H} \cdots A$ \\
\hline $\mathrm{N} 1-\mathrm{H} 1 N^{\cdots} \cdots 1^{\mathrm{i}}$ & 0.88 & 2.13 & $2.871(3)$ & 142 \\
$\mathrm{~N} 4-\mathrm{H} 4 N \cdots \mathrm{O} 4^{\text {ii }}$ & 0.88 & 2.04 & $2.794(3)$ & 142 \\
$\mathrm{C} 4-\mathrm{H} 4 B \cdots 6^{\text {iii }}$ & 0.98 & 2.54 & $3.075(4)$ & 114
\end{tabular}


supporting information

$\begin{array}{llllr}\mathrm{C} 6-\mathrm{H} 6 B \cdots \mathrm{O} 2^{\text {iv }} & 0.98 & 2.60 & 3.518(4) & 156 \\ \mathrm{C} 7-\mathrm{H} 7 B^{\cdots}{ }^{\text {iv }} & 0.98 & 2.52 & 3.431(4) & 155 \\ \mathrm{C} 11-\mathrm{H} 11 B \cdots \mathrm{O} 3^{\mathrm{v}} & 0.98 & 2.53 & 3.042(4) & 113 \\ \mathrm{C} 13-\mathrm{H} 13 B \cdots \mathrm{O} 4^{\text {iv }} & 0.98 & 2.53 & 3.440(4) & 155 \\ \mathrm{C} 14-\mathrm{H} 14 B \cdots 5^{\text {iv }} & 0.98 & 2.59 & 3.554(4) & 168\end{array}$

Symmetry codes: (i) $x-1 / 2,-y, z$; (ii) $x-1 / 2,-y+1, z$; (iii) $-x+1,-y+1, z+1 / 2$; (iv) $x-1, y, z$; (v) $-x+1,-y, z-1 / 2$. 